Obstáculos al implantar el modelo CMMI

Difficulties when implementing the CMMI organizational model

Dbstacles à la mise en place du modèle LMMI

Dbstáculos na implantą̧ãa do modela cmmi
FECHA DE RECEPCIÓN: 18 de febrero FECHA DE APROBACIÓN: 12 de abril pp. 110-127

\section{Himelda Palacios*}

Néstor Porcell**
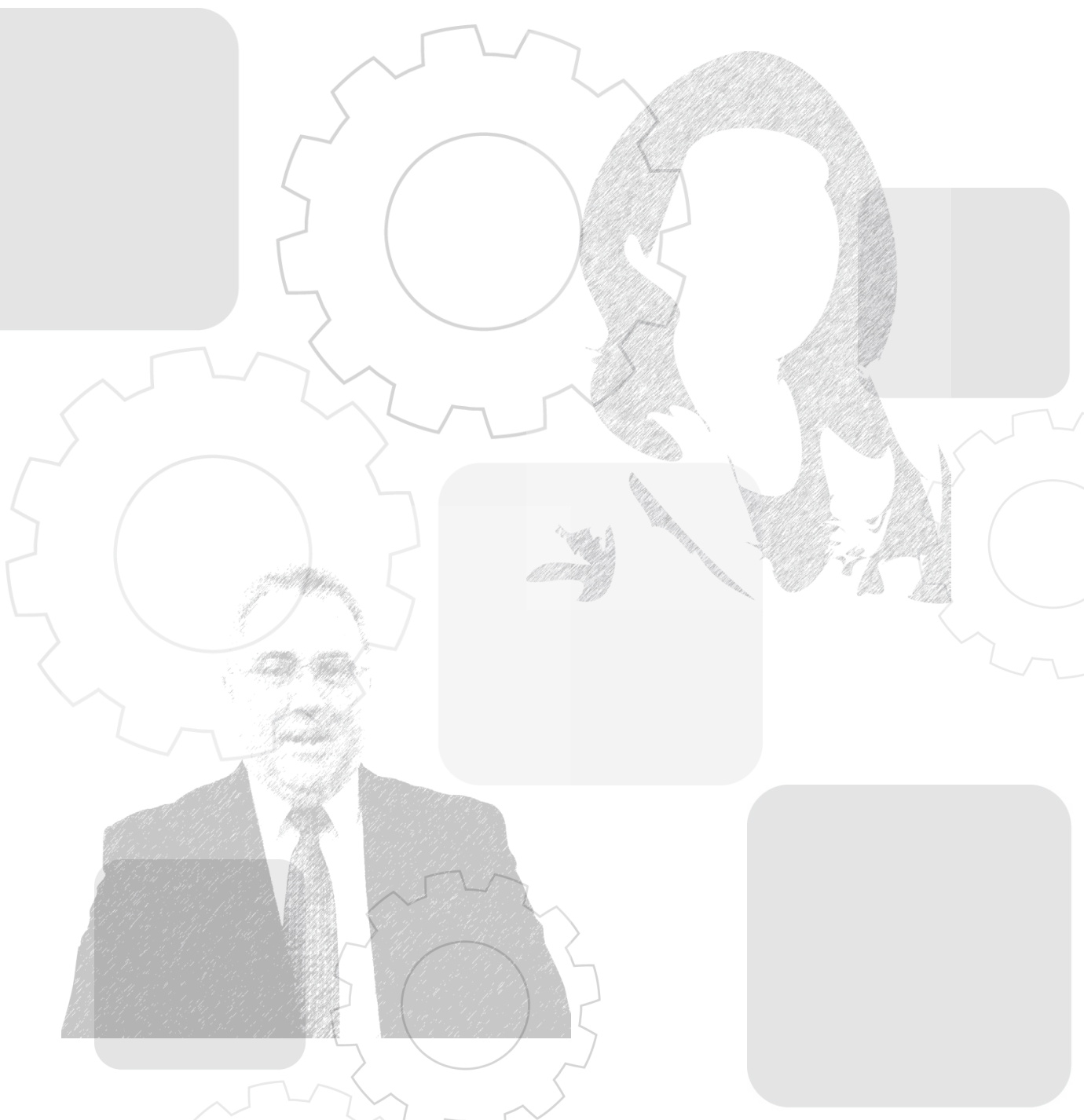

* Maestría en Ingeniería Industrial, Universidad de Los Andes;

Especialización en Banca, Universidad de Los Andes;

Especialización en construcción de Software, Universidad de Los Andes; Ingeniera de Sistemas y Computación, Universidad de Los Andes.

** Ingeniero Industrial con Maestría en Ingeniería de la Universidad de Puerto Rico con énfasis en Sistemas de Manufactura, Decano Facultad de Ingeniería Universidad EAN 


\section{RESUMEN}

Esta investigación analiza y propone estrategias de intervención que permiten identificar y superar los obstáculos de aprendizaje organizacional, que experimenta una organización cuando decide implantar el modelo de calidad para el desarrollo y mantenimiento de software, CMMI (Capability Maturity Model Integrated). Implantar el CMMI es más que definir procesos, procedimientos y formatos, lo que realmente implica es cambiar la cultura organizacional de las áreas y/o empresas de desarrollo de software, cambiar el comportamiento de los Ingenieros de Software.

Las estrategias de intervención propuestas facilitan el cambio de la cultura organizacional requerido, para que una organización dedicada al desarrollo y mantenimiento de software pueda alcanzar con éxito los niveles de madurez definidos por el modelo CMMI. Este cambio de cultura implica orientar a la organización hacia los lineamientos definidos por la gestión de la calidad del software, la ingeniería de software, la gerencia de proyectos, la gerencia de procesos, el mejoramiento continuo de procesos, la gestión cuantitativa de procesos y el aprendizaje continuo.

\section{ABSTRACT}

This research aims at analysing and proposing intervention strategies which allow to identify and avoid the risks in organization learning processes, when an organization decides to implement a quality model for the development and maintenance of specialized softwares, such as CMMI (Capability Maturity Model Integrated) - This software design means much more than identifying processes, procedures and formats, which implies an organizational cultural change in the corresponding areas and companies in charge of software development changing the behavior of software design engineers.

The intervention strategies suggested facilitate a change in organizational culture in this type of organization reaching success at high maturity levels based on the CMMI model. This will lead the organization to high quality standards in software design, software engineering, project management, process management, a continuing successful follow- up processes, quantitative process management and systematic learning.

\section{RESUMÉÉ}
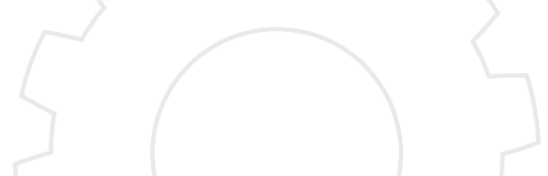

Cette étude analyse et propose différentes stratégies d'intervention aidant à l'identification et à la résolution de problèmes liés à l'apprentissage organisationnel. Cela peut être le cas pour une organisation qui déciderait de mettre en place le modèle de qualité pour le développement et la maintenance de logiciels, CMMI (Capability Maturity Model Integrated). En effet, pour intégrer le CMMI aux processus organisationnels de l'entreprise, il s'agit moins de définir les procédures, formats et processus que de modifier la culture organisationnelle des départements et/ou entreprises informatiques de développement de logiciel ainsi que le comportement des ingénieurs informatiques.

Les stratégies d'intervention proposées favorisent le changement de culture organisationnelle nécessaire pour qu'une organisation, tournée vers le développement et la maintenance de logiciel, puisse atteindre avec succès des niveaux de maturité définis par le modèle CMMI. Ce changement culturel implique une orientation de l'organisation aux directives définies par la gestion qualitative des logiciels, la gestion de projet, celle des processus de gestion, de l'amélioration continue des processus, de la gestion quantitative des processus et de l'apprentissage continu.

\section{RESUMO}

Esta pesquisa analisa e propõe estratégias de intervenção que permitem identificar e superar os obstáculos de aprendizado organizativo experimentados por uma organização quando decidir implantar o modelo de qualidade para o desenvolvimento e manutenção de software, CMMI (Capability Maturity Model Integrated). Implantar o CMMI é mais do que definir processos, procedimentos e formulários; em realidade implica a mudança da cultura organizativa das áreas elou empresas de desenvolvimento de software, mudar o comportamento dos engenheiros de software.

As estratégias de intervenção propostas facilitam a mudança da cultura organizativa requerida, para que uma organização dedicada ao desenvolvimento e manutenção de software possa atingir com sucesso os níveis de maturidade definidos pelo modelo CMMI. Esta mudança de cultura implica orientar a organização em direção aos lineamentos definidos pela gestão da qualidade do software, a engenharia de software, a gerência de projectos, a gerência de processos, o melhoramento contínuo de processos, a gestão quantitativa de processos e o aprendizado contínuo.

\section{Palabras claves}

CMMI

Gestión de la calidad

Gerencia de procesos

Aprendizaje continuo

\section{Key words}

CMMI model

Quality management

Process management

Systematic learning

\section{Mots clefs}

\section{CMMI}

Gestion de la qualité

Processus de gestion

Apprentissage continu

\section{Palavras-chave}

\section{CMMI}

Gestão da qualidade

Gerência de processos

Aprendizado contínuo 


\section{APRENDIZAJE ORGANZACIONAL}

aprendizaje organizacional es el proceso mediante

el cual la organización incrementa su capacidad de acción (Espejo, Schuhmann, Schwaninger y Bilello, 1996). La esencia del aprendizaje es la habilidad de adaptarse al cambio, y para implantar el modelo

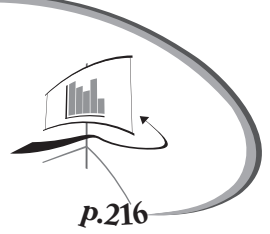

CMMI, es necesario efectuar cambios en políticas, valores y rutinas de trabajo de una organización. Cuando se desarrolla un aprendizaje organizacional, las organizaciones aprenden a hacer mejor lo que hacen, y simultáneamente aprenden a cuestionar sus propias políticas, normas, valores y prácticas. Una ororganización aprende a través del aprendizaje de sus individuos, sin embargo, estos pueden aprender sin que sus organizaciones lo hagan, ya que las rutinas que siguen pueden no ser afectadas por el conocimiento que tienen en sus mentes.

El aprendizaje individual incluye la adquisición de conocimiento y habilidades, en consecuencia se da en dos dominios de acción (Espejo, et al., 1996):

- Aprendizaje conceptual: relacionado con conocer el porqué, es decir, la capacidad de entender la experiencia y la percepción.

- Aprendizaje operacional: relacionado con conocer el cómo, es decir, la capacidad de producir una situación deseada.

En consecuencia, el aprendizaje tiene dos significados asociados, lo que se aprende y como lo aprendido es entendido, interpretado y utilizado.

De acuerdo con Kofman (1992), el proceso de aprendizaje se puede describir como un ciclo OADI ( Observe, Asses, Design, Implement). Este ciclo se puede entender así: de la observación de eventos o experiencias específicas se realizan evaluaciones. De estas reflexiones se derivan hipótesis o conclusiones, las cuales dan lugar al diseño de modelos conceptuales abstractos. Cuando estos modelos son implementados, son probados en la realidad, y el ciclo de aprendizaje se inicia nuevamente, con la observación de los resultados del experimento.

De igual forma sucede al implantar una práctica del CMMI, inicialmente se observan unos problemas, por ejemplo, cronogramas atrasados e irreales, se analiza y reflexiona sobre métodos de estimación de esfuerzo y se diseña un procedimiento de aplicación de un método particular. Luego este se aplica a un proyecto piloto real, para probar su

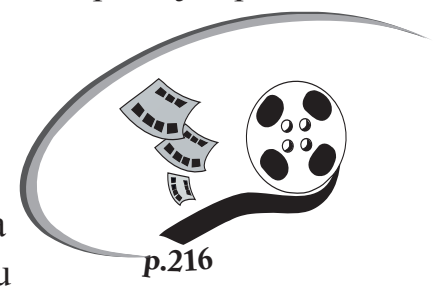
efectividad y determinar posibles ajustes, los cuales, una vez realizados se implantan al resto de la organización. La aplicación reiterada del ciclo OADI, a cada una de las prácticas del CMMI, es lo que en suma, permite la implantación en un área de proceso.

Los dos conceptos anteriores están estrechamente ligados. El aprendizaje operacional está asociado con las etapas de observar implementar (Espejo, et al., 1996) del ciclo OADI. Esto se debe a que los pasos para hacer un trabajo, son lo que constituyen las rutinas diarias de operación. Complementariamente, el aprendizaje conceptual está asociado con las etapas de evaluar y diseñar del OADI. Este es el aprendizaje requerido por un individuo para construir y adaptar sus modelos mentales a las rutinas diarias, lo cual incluye entender las razones de porque son necesarios dichas rutinas y procedimientos.

Por último, el aprendizaje tanto individual como organizacional, puede ser de dos tipos (Espejo, et al., 1996):

- Aprendizaje de circuito simple: este aprendizaje ocurre cuando se detectan errores y estos son corregidos dentro del marco presente y aceptable de normas, valores y metas.

- Aprendizaje de circuito doble: este aprendizaje ocurre cuando, además de detectar y corregir errores, hay un cuestionamiento y modificación de las normas, valores o metas de la situación correspondiente. 

Figura 1. Modelo CMMI

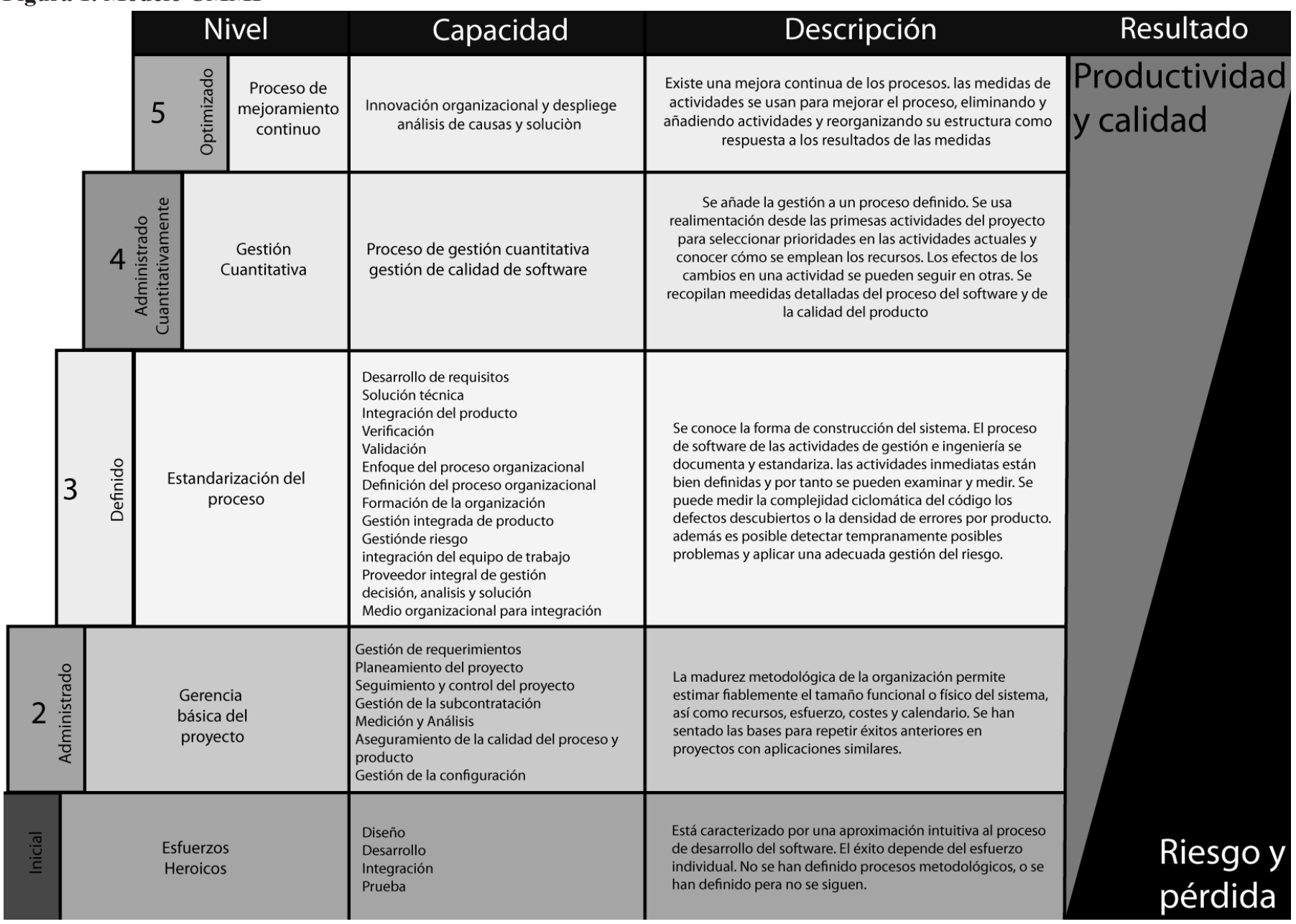

Fuente. SEI Carnegie Mellon.

A continuación, se describen los dos primeros niveles, objeto de esta investigación.

\subsection{Nivel 1 de madurez: inicial}

En el nivel inicial no existen procesos o son caóticos y no se ejecutan de forma recurrente y disciplinada. La organización no provee un ambiente estable que de soporte a los procesos. El éxito en las organizaciones de nivel 1 depende de la competencia y actos heroicos de las personas, y no de sus procesos productivos. Aunque la organización crea productos y servicios, estos con frecuencia exceden sus presupuestos y no satisfacen sus cronogramas y requerimientos funcionales del cliente. Las organizaciones de nivel 1 se caracterizan por una tendencia a comprometerse excesivamente, más allá de su capacidad, abandonar sus procesos productivos durante los periodos de crisis, no gestionar su conocimiento, tener alta rotación de los ingenieros de software, ejecutar los proyectos sin planearlos, no documentar, vivir apagando incendios, y con una incapacidad para repetir sus éxitos.

\subsection{Nivel 2 de madurez: administrado}

En el nivel 2 de madurez, los equipos de proyecto de la organización se han asegurado de administrar los requerimientos funcionales del proyecto, y los procesos productivos son estimados, planeados, entrenados, realizados, medidos, controlados, verificados y mejorados continuamente. La disciplina de procesos establecida por el nivel 2 ayuda a 




\section{RETUS DEL CAMBID PROFUNDD}

I

ara la gran mayoría de los líderes empresariales, la única ventaja competitiva real que tendrá su compañía, reside en la capacidad de sus directivos para aprender más rápido que sus competidores. Lo cual se ve incrementado en nuestro siglo por la globalización, el Internet, la integración de mercados y el avanzado desarrollo tecnológico. Sin embargo, algunas empresas

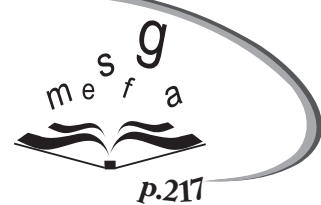

p.217 en Colombia, aun se resisten tanto a mirar el resto del mundo, como a aceptar que lo único constante es el cambio, lo cual ha sido una realidad de la vida humana, por lo menos desde los tiempos de Heráclito (c. 500 a.de J.C.) Es por esto, que es crucial gestionar el aprendizaje en nuestras empresas.

Peter Senge, en su libro "La danza del cambio", propone una serie de retos u obstáculos que inhiben el aprendizaje en una organización. Estos retos se basan en el concepto de cambio profundo, el cual está definido como el "cambio organizacional que combina una modificación interna en los comportamientos, valores, y aspiraciones de las personas; con una modificación externa en las estrategias, procesos, prácticas y sistemas" (Senge, 1999). Es importante resaltar que todo cambio profundo involucra un aprendizaje organizacional, y sostener un proceso de cambio profundo requiere una modificación fundamental de nuestra manera de pensar y de actuar. Igualmente, el aprendizaje ocurre en el tiempo y en la vida real, no en un salón de clase, ni en sesiones de capacitación.

Senge ha identificado diez retos, clasificados en retos de iniciar el cambio, sostenerlo y rediseño. A continuación se describe cada uno de ellos. Senge, (1999).

\subsection{Retos de la iniciación}

Surgen cuando cualquier grupo de innovación, en nuestro caso el Engineering Process Group (EPG) o los Process Action Teams (PATs), inician un proceso de cambio, y empiezan a hablar y a llevar a cabo su trabajo en formas distintas al resto de la organización. Está compuesto por 4 retos:

- Falta de tiempo suficiente: es el reto de control y gestión de nuestro tiempo. Los que toman parte en una iniciativa de cambio necesitan suficiente flexibilidad para dedicar tiempo a la reflexión, evaluación y práctica.

- Falta de soporte y ayuda: este es el reto de un entrenamiento, guía y apoyo inadecuado para los grupos de innovación, además, del reto de desarrollar recursos internos para crear una capacidad de aprender.

- No relevancia: el reto de lo que es improcedente, defender el cambio planteando claramente una justificación y un enfoque apropiado para el negocio y mostrando por qué al desarrollar capacidades de aprendizaje, estas son pertinentes para las metas comerciales de la empresa.

- Cumplir lo que se promete: el reto de la claridad y las consecuencias de la administración, la incongruencia entre la conducta y los valores que se defienden.

\subsection{Retos de sostener el cambio}

Ocurren dentro de los grupos de innovación (EPG, PATs, grupos pilotos), cuando alcanzan un éxito temprano, y no pueden seguir actuando de manera aislada de la organización. Estos retos son (Senge, 1999): 
- Temor y ansiedad: el reto del temor a la exposición y crítica, la vulnerabilidad e incapacidad, iniciados por el conflicto entre niveles crecientes de franqueza y apertura, y bajos niveles de confianza ente los miembros de los grupos de innovación.

- Evaluación y medición: este reto se presenta cuando los grupos de innovación interactúan con el resto de la organización. Es el reto de la evaluación negativa del progreso del proyecto, causado por la desconexión entre las maneras tradicionales de medir el éxito (tanto las mediciones como el horizonte de tiempo) y las nuevas prácticas definidas y aplicadas por los grupos de innovación.

- Verdaderos creyentes e incrédulos: corresponde al reto del aislamiento y arrogancia que aparece cuando los verdaderos creyentes dentro de los grupos de innovación, se enfrentan a los incrédulos que están fuera de dichos grupos. Constantemente se interpretan mal unos y otros. Este reto se presenta cuando los grupos de innovación interactúan con el resto de la organización.

\subsection{Retos de re- pensar y re-diseñar}

Aparecen cuando la iniciativa de cambio alcanza más amplia credibilidad y se enfrenta a la estructura interna y a las prácticas habituales de la organización. Estos retos son (Senge, 1999):

- Gobierno: es el reto de la estructura existente de gobierno y el conflicto entre los grupos de innovación, que buscan mayor autonomía, y los directivos que se preocupan de que esta pueda llevar al caos y a la fragmentación interna.

- Difusión: este reto se relaciona con la transferencia del conocimiento a las diferentes unidades organizacionales, dificultando así que los que pertenecen a una misma unidad aprovechen recíprocamente los éxitos que otros obtienen.

- Estrategia y propósito: es el reto de cuestionar hacia dónde va la organización, revitalizar y repensar los objetivos, su identidad y sus estrategias.

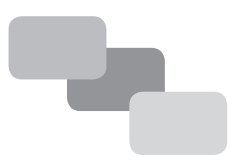

\section{GESTIÍN DE UN PRDYECTD DE IMPLEMENTACIÍN DEL CMMI}

1 no de los errores típicos que cometen las organizaciones de nivel 1 , cuando van a implantar el nivel 2 , es no considerar esta iniciativa como un proyecto, al cual es necesario aplicarle todas las prácticas de gestión de proyectos. Esto lo que significa es que el EPG debe aplicar el nivel 2 para la implantación, antes de que la empresa inicie la iniciativa de cambio. Por lo general, el comportamiento del grupo del EPG debe ir un nivel de madurez adelante, del nivel que se pretende implantar. De lo que se trata en este caso, es de convencer con el ejemplo, y transmitir confianza a la organización en el sentido de que las prácticas del nivel 2 son realizables en la empresa.

Es bastante difícil para el EPG convencer y/o exigir a los equipos de desarrollo de software de la empresa, que estimen el tamaño y esfuerzo, usen planes para sus proyectos, manejen riesgos, controlen las versiones, monitoreen el progreso y tomen acciones correctivas, sigan unos estándares dados y midan su desempeño, cuando el EPG es el primero en no aplicar estas mismas prácticas, para la implantación del CMMI. 
Los aspectos claves a tener en cuenta en este sentido son los siguientes:

- Definición clara del alcance del proyecto, usando un Work Break-down Structure (WBS).

- Definición y establecimiento de la infraestructura de mejora, indicando los roles, responsabilidades, dedicación, y entregables a generar, para el Managing Steering Group (MSG), EPG, PATs, grupo PPQA (Process and Product Quality Assurance) y el Grupo de Implantación de Procesos (GIP).

- Definir una estrategia de implantación, basada en los objetivos de negocio.

- Planear el proyecto. Como mínimo, elaborar el plan de acción de mejoramiento, para todo el proyecto, y los planes de acción de mejora para cada uno de los PATs. La elaboración del primero, fácilmente puede tomar una semana completa. Solo contra estos planes es que se podrá informar a la empresa el progreso del proyecto.

- Es necesario definir el esquema y la periodicidad del monitoreo y control del proyecto. Para generar confianza, todos deben saber cuánto se ha avanzado.
- Es crítico identificar y gestionar los riesgos del proyecto, pues son muchas las fuerzas y eventos que presionan e inhiben el cambio de prácticas y de comportamiento. Sin esto, la iniciativa puede fracasar más rápido. Crear y administrar un depósito con control de versiones y de cambios, de los nuevos procesos de la empresa, que sea de fácil acceso y consulta para todos los empleados de la organización.

- Establecer un plan de incentivos, para motivar el cambio a las nuevas prácticas.

- Definir y planear todo el entrenamiento requerido en la organización, tanto en el modelo y sus procesos, como en las nuevas prácticas de la empresa. El cambio no es gratuito y no se genera enviando el típico correo electrónico con el texto de "a partir del próximo lunes, todos los proyectos usaran el formato de...".

- Generar espacios para la autocrítica, recolección de propuestas de mejora y retroalimentación de los cambios propuestos. 


\section{Б. DBSTÁCULLS DE APRENDIZAJE EN EL CMMI Y ESTRATEEIAS DE SDLUCIÓN}

as capacidades de aprendizaje del CMMI no pueden ser forzadas, ni apresuradas, ni impuestas por otro. La frase de tenemos 12 meses para implantar el nivel 2, aunque posible con muchísimo esfuerzo y dedicación, por lo general no es alcanzable. El aprendizaje necesita tiempo, pues no es solo definir nuevos procesos, sino aplicarlos en el día a día. Tampoco se trata de copiar y pegar procedimientos y formatos de otros. Usando una metáfora, implantar el CMMI es similar a adelgazar, crear un equipo para jugar al tenis, o aprender a bailar. Podemos leer muchos libros al respecto, ver muchos videos de cómo hacerlo, tener un entrenador personal que nos guíe, pero solo cada uno de nosotros puede hacerlo y coordinarse con los demás, porque debe hacerse mediante el trabajo en equipo. Este proceso de crecimiento y aprendizaje se da a nivel de los resultados personales, los resultados de negocios y la creación de redes de personas comprometidas con el cambio.

A continuación se presenta el análisis de los 10 retos al implantar el CMMI y algunas guías de cómo vencer estos obstáculos.

\subsection{Retos de la iniciación}

\subsubsection{Falta de tiempo suficiente}

La implantación del CMMI debe hacerse en paralelo con los proyectos de software de empresa, con las mismas personas y durante el tiempo laboral. Las iniciativas de trabajamos en CMMI después de las 6, o el fin de semana son garantía de fracaso, porque van en contra de los valores que el CMMI propone. A medida que se hace más fuerte la inversión en la iniciativa de cambio, aumenta la percepción de la gerencia, los PATs y los equipos de proyecto, del tiempo requerido para participar en la definición e implantación de las nuevas prácticas. Si es baja la flexibilidad y disponibilidad de tiempo de las personas, porque ya están comprometidas de tiempo completo en los demás proyectos de la empresa, no se comprometerán con las tareas del proyecto CMMI y se llegará a un vacío de tiempo. Esto ocurre por dos razones: o bien el tiempo que se invierte es ineficaz, porque las personas claves faltan a las reuniones importantes, o bien las personas no se comprometen porque sienten frustración, porque les parece que no hay tiempo suficiente para ese proyecto, porque la prioridad son los clientes.

La solución a este reto es determinar el impacto del proyecto en las labores del día a día, y elaborar el plan de acción de mejora que detalle todas las fases y actividades requeridas en el proyecto, a fin de determinar las necesidades reales de tiempo y recursos en la organización, para así, hacer una redistribución de actividades. Esto puede implicar la contratación de personas temporales, a las cuales se les pueda delegar actividades del día a día, para que los grupos de innovación puedan hacer unos planes realistas y comprometerse con la iniciativa.

\subsubsection{Falta de soporte y ayuda}

Debido a las falencias de los Ingenieros en el conocimiento y uso de técnicas y metodologías de Ingeniería de Software, este proyecto requiere de un fuerte entrenamiento, guía y apoyo adecuado para los grupos de innovación. El primer cambio notorio se da en el lenguaje, pues aparecen un sinnúmero de nuevos conceptos. Las personas leen las prácticas, pero desconocen de qué se trata. Esta situación se empeora por el idioma Inglés, pues muchas personas lo desconocen y el primer obstáculo es la lectura. Si el apoyo del que disponen las personas es inadecuado, se genera un vacío de ayuda y la eficacia de la iniciativa se perjudica, impidiendo el desarrollo de las capacidades de aprendizaje. Las frases típicas son "no entendí, ni para preguntar", o "no sabemos qué hacer". La frustración enfriará el entusiasmo de todos y su voluntad de comprometerse y entonces, la iniciativa de cambio se retarda o muere lentamente. 
Hay dos atenuantes adicionales, en algunas empresas el no saber es sinónimo de incompetencia y la mayoría de los ingenieros tienen un ego enorme que les hace creer que todo lo pueden hacer solos. Además, algunos creen en la magia, y piensan que con instalar herramientas automatizadas de apoyo, con eso el proceso CMMI quedará instantáneamente implantado.

La solución a este reto es invertir temprano en ayuda, contratando asesores expertos, que tengan el conocimiento conceptual y operacional del modelo, comprobado en otras empresas. Es frecuente que algunos asesores transcriben conceptos de Ingeniería de Software a diapositivas de capacitación, pero cuando las personas preguntan cómo se haría en su empresa, no tienen una respuesta concreta. El CMMI solo define el que, y es tarea de la organización diseñar el cómo. Por eso, es necesario capacitar a la gerencia, al EPG, y a los PATs en el modelo, en los procesos específicos a implantar y contar con una librería de conceptos y ejemplos de procesos. Esto facilitará la definición de procesos.

Además, es necesario identificar y planear la ayuda requerida en cada fase del modelo IDEAL. Por ejemplo, la organización deberá aprender sobre diagnósticos, planes de acción de mejora, entre otros. Paralelamente, es necesario disponer de un diccionario de términos de fácil acceso y consulta, pues el lenguaje en la organización cambiará y si no es claro para todos, su significado y alcance, generará graves problemas de institucionalización de procesos, que le impedirán a la empresa alcanzar un nivel de madurez.

Similarmente, una estrategia clave, durante las etapas de definición y de implantación de los procesos, es utilizar modelos conceptuales (Checkland, 1981) para los procesos del CMMI. El modelo es extenso, y un diagrama claro y conciso vale más que mil palabras. Una vez definido el proceso, se recomienda seleccionar y hacer uso de herramientas automatizadas de apoyo al proceso, para facilitar el aprendizaje operacional y la institucionalización de las prácticas. Además, durante la etapa de implantación y de aprendizaje del IDEAL, es necesario crear en el EPG una capacidad de soporte para la solución de dudas de proceso, con mecanismos de atenuación, como monitores, preguntas frecuentes, y foros de discusión.

Por último, es necesario desarrollar recursos internos para crear una capacidad de entrenamiento autónoma, es decir, crear cursos, material de capacitación, grupos de investigación y personal de apoyo para entrenar a la organización en temas particulares y de forma continua. Es igual que un niño, el cual debe caminar por sí solo, y no todo el tiempo de la mano de un adulto, pues aunque es probable que al inicio la empresa asigne recursos para asesores externos, la empresa debe crear su autonomía.

\subsubsection{No relevancia}

Afrontar este reto implica establecer un argumento claro, auténtico y persuasivo sobre la pertinencia de implantar CMMI en la empresa, tanto para el bienestar del negocio como para los empleados, ellos deben saber como el CMMI los beneficiará en sus labores del día a día. El requisito clave de este reto es el compromiso, por eso cada persona de todos los niveles organizacionales se debe poder conectar personalmente con el modelo. Para esto las personas necesitan ver que este cambio está enfocado a las necesidades claves del negocio y necesitan entender cómo encajan ellos, cómo pueden contribuir y cómo se beneficiarán. Si no se satisfacen estas necesidades, se produce un vacío de compromiso y en sus mentes se preguntarán ¿Por qué estamos haciendo esto? El riesgo es que participarán porque es obligatorio, no porque crean que sea definitivo para el éxito de la empresa.

Algunas soluciones a este reto son: alinear los objetivos del proyecto CMMI, con los objetivos del negocio, entender la visión del modelo y en particular la visión del nivel 2, elaborar una justificación del proyecto, definir los beneficios de cada proceso y las consecuencias en el desempeño personal y de la organización, de su no realización y comunicar estas aspectos a todas las personas. Suele suceder que la alta gerencia hace este ejercicio, pero la gerencia media no lo comunica a sus equipos de trabajo. Por último, y basado en la experiencia de los autores en este campo, la responsabilidad del cambio no debe darse al EPG, que solo es un grupo coordinador y facilitador. En lugar de eso, se recomienda establecer un Grupo de Implantación de Procesos (GIP), responsable de la institucionalización y aprendizaje conceptual y operacional de los procesos CMMI en los proyectos de la organización. 


\subsubsection{Cumplir lo que se promete}

En este reto el aspecto clave está en la congruencia entre el lenguaje de la gerencia, sus valores y su comportamiento. Se da cuando los valores expuestos son diferentes a los valores aplicados. Este reto tiene dos manifestaciones. Cuando las personas escuchan declaraciones de la gerencia del estilo: de ahora en adelante, todo proyecto será estimado con detalle y tendrá su plan completo; pero cuando llega el proyecto urgente o el cliente estratégico, la gerencia dice empiece a programar, porque de eso es que comemos; inmediatamente se genera un vacío de confianza y de valores, que eliminará la credibilidad de las personas en la iniciativa de mejora y pensarán que los negocios se seguirán haciendo como siempre, sin importar los nuevos procedimientos creados por los PATs. La segunda manifestación del reto se da en la falta de confianza que sienten las personas al exponer los problemas de la empresa, cuando reflexionan sobre el comportamiento actual frente al esperado con las nuevas prácticas del modelo.

Estas reflexiones a menudo surgen en ambientes grupales, en los PATs, sesiones de entrenamiento, grupos pilotos, entre otros. Cuando los niveles gerenciales atacan estas reflexiones, o, justifican el comportamiento habitual, las personas perciben que no podrán confiar en que sus líderes apoyarán los nuevos valores y procesos. En consecuencia, se produce un vacío de reflexión sobre el cómo aplicar el CMMI, el cual disminuirá el deseo de comprometer tiempo $\mathrm{y}$ esfuerzo a investigar, proponer soluciones y tomar riesgos, haciendo pilotos de estas.

Algunas soluciones a este reto son: educar a la gerencia en sus futuras y nuevas responsabilidades, definir la cultura organizacional que se quiere establecer con el consenso de la gerencia y crear credibilidad de valores y propósitos por demostración en las actividades diarias. Nada convence más que el ejemplo. Una buena estrategia en estos casos es hacer una actividad de auto-evaluación al final del proyecto, en la cual se identifique que se hizo bien, que se hizo mal, se documenten las lecciones aprendidas y se tomen las acciones correctivas necesarias. Es clave compartir estas lecciones con todas las personas, en especial con los escépticos, de esta forma se genera confianza de que el cambio es real y es posible.

\subsection{Retos de sostener el cambio}

\subsubsection{Temor y ansiedad}

Por lo general las personas se acostumbran a trabajar como en silos, concentrados en su área funcional e ignorando la interacción entre las demás áreas, a pesar de que el desarrollo de software es un trabajo en equipo. A medida que se desarrollan las capacidades de aprendizaje del modelo, las personas (iniciando en los PATs) comienzan a sentir confianza para proponer cambios de fondo y discutir problemas de operación que son retantes e incluso conflictivos, frente a las prácticas actuales. Pero otras personas se sienten un poco amenazadas con estos cambios y discusiones, pues aunque no les guste por completo la forma tradicional de trabajar, se sienten seguros y saben cómo manejar las situaciones. Como ahora no saben que esperar con los cambios, esta incertidumbre genera miedo y ansiedad, que por lo general se manifiesta en forma de rechazo a los cambios propuestos. Esta situación se torna crítica, cuando son las posiciones gerenciales las que exhiben el rechazo. Sin embargo, es importante reconocer que estos sentimientos son naturales en todo proceso de cambio.

El aspecto clave que el EPG debe trabajar aquí, es la generación de un ambiente de seguridad y confianza sicológica a todos los niveles de la organización, con el cual debe construir una capacidad de apertura tanto a nivel individual como colectivo. La apertura debe darse tanto para sacar a la luz los problemas, como también para escuchar abiertamente las soluciones propuestas y reconocer la existencia de diferentes puntos de vista. Si quienes proponen los cambios no tienen estos niveles de confianza, se generará una brecha de apertura, la cual genera miedo y ansiedad, sentimientos que hacen que el entusiasmo y la voluntad de comprometerse con la definición y uso de las nuevas prácticas disminuya, con lo cual se reduce la inversión en tiempo y dinero en la iniciativa de cambio, reducción que a su vez, va restringiendo las capacidades de aprendizaje individual y colectivas, que son la clave para mejorar los resultados personales y de negocio.

Algunas soluciones a este reto son: realizar cambios pequeños para fomentar la confianza en la empresa, iniciar con logros tempranos para crear la convicción y seguridad colectiva para implantar el CMMI. Además, definir los 
procesos en forma incremental e iterativa, para aumentar los niveles de seguridad psicológica y apertura en los diferentes grupos de trabajo. Desde el comienzo, instaurar una visión compartida de los procesos del modelo, mediante el uso de los modelos conceptuales. Por último, y para la etapa de implantación de los cambios, crear en el EPG un equipo de soporte permanente, apoyado con un esquema automatizado de preguntas frecuentes, mediante el cual se responda a todas las dudas que surgen cuando los equipos de proyectos comienzan a utilizar los nuevos procesos.

\subsubsection{Evaluación y medición}

Este reto se relaciona con la forma como las personas y la organización juzga si las nuevas prácticas están funcionando y mejorando la calidad del software. Los nuevos procesos pueden contradecir la manera de pensar utilizada para calcular las mediciones e indicadores actuales. El comentario que por lo general se escucha es: antes hacíamos estos proyectos en ocho días y ahora nos toma el doble del tiempo. Todos olvidan de inmediato, las incontables horas dedicadas a la corrección de errores y ajustes de última hora, después de la entrega del software. Permitir estos comentarios y continuar con los antiguos indicadores distorsionarán y entorpecerán la implantación del CMMI y el cambio de cultura. La evaluación del éxito de los cambios es un aspecto complejo y ambiguo. Además, cuando se implanta el proceso de Medición y Análisis, del nivel 2, la empresa debe definir las mediciones y analizar el desempeño de los nuevos procesos.

Otro aspecto en este reto es que poco después de empezar los cambios de prácticas, muchos esperan ver una mejora en los resultados comerciales. Sin embargo, hay demoras significativas en la implantación y es importante tener en cuenta las estadísticas existentes, para no generar falsas expectativas. La implantación del CMMI no es una iniciativa del corto plazo. Si el horizonte de tiempo implícito en la mente de las personas es corto, las expectativas por resultados son altas y se genera un vacio inicial de resultados del negocio, entre lo que se esperaba y lo que en realidad se ha obtenido, el cual da lugar a evaluaciones negativas dentro de la empresa. Si las mejoras observadas están muy a la zaga de lo que se esperaba, los PATs y los equipos de implantación empiezan a perder credibilidad, y se quedan sin argumentos para justificar el tiempo que dedican a las nuevas prácticas, con lo cual disminuye su grado de compromiso y dedicación al cambio, y en consecuencia se reducen las capacidades de aprendizaje.

Algunas soluciones a este reto son: definir el horizonte de tiempo para obtener los resultados del proyecto, al momento de hacer su lanzamiento apreciar y reconocer los tiempos de espera, implementar los objetivos y prácticas genéricas, usar el método de Goal Question Metric (GQM) (Kan, 2003), definir nuevos indicadores de éxito para los proyectos de software, y efectuar retro-alimentación de las métricas a toda la organización. EPG, mediante los indicadores, debe hacer visible el avance en los niveles de madurez de la organización.

\subsubsection{Verdaderos creyentes e incrédulos}

En este reto hay cuatro ciclos limitantes. El primero se refiere a las amenazas percibidas por las personas que no hacen parte de los grupos de innovación, con relación a nuevas prácticas o conductas y mejores resultados de negocios, las cuales desconocen y critican negativamente. El segundo está compuesto por las personas que quieren participar en los cambios, pero que cuando quieren entender se enfrentan a unas teorías y una jerga o lenguaje que nadie les explica claramente, y en consecuencia terminan siendo aislados y vistos como grupos de innovación incomprendidos. El tercer caso es cuando los grupos de innovación se colocan a la defensiva y no responden eficazmente a las preguntas del resto de la organización, perdiendo credibilidad ante la empresa. El último caso se da cuando los grupos de innovación tienen éxito, mejoran sus resultados de negocio y adquieren tal confianza que se vuelven arrogantes y son incapaces de atraer e inspirar al resto de la organización hacia el cambio.

En todos los casos, los aspectos críticos son la falta de una flexibilidad cultural ante la diversidad de prácticas y un vacío de participación entre los grupos de innovación y el resto de la organización que combinados disminuyen la voluntad de comprometerse con los cambios.

Algunas soluciones a este reto son: hacer el lanzamiento del proyecto a toda la organización, pues la implantación del CMMI afecta a todas las personas. Por eso, el EPG debe comunicar el plan de implantación e informar 
periódicamente a la organización, los logros alcanzados y el estado de avance del proyecto. Para que no haya mal entendidos, todos deben saber que es lo que está pasando, y como los cambios los afectan y benefician.

\subsection{Retos de re-diseñar y re-pensar el cambio}

\subsubsection{Gobierno}

Este reto suele presentarse mediante choques de las posiciones gerenciales con los grupos de innovación (EPG, PATs y Pilotos), causados por la autonomía y el sentido de poder que estos desarrollan, cuando definen las nuevas prácticas de la empresa, pues en suma, son ellos los que están redefiniendo la empresa. La poca tolerancia de los niveles gerenciales por esta independencia, se manifiesta imponiendo controles administrativos que no generan valor a la implantación del CMMI, porque piensan que los grupos de innovación no entienden el contexto general del negocio, ni el impacto estratégico de las decisiones locales que están tomando. Para ambos grupos, las consecuencias son una reducción en el compromiso frente a los cambios.

Otro aspecto de este reto, es el relacionado con el diseño del proceso y su integración con las diferentes unidades de negocio, ya que si el grupo de innovación no tiene la habilidad para manejar la interdependencia entre procesos y unidades, surge un vacío de integración. Cuando este reto sucede, el grupo de innovación se encuentra en desacuerdo con otros grupos y con la alta dirección, lo que da como resultado un aumento en el control administrativo y una baja del compromiso, lo cual genera una disminución en tiempo y recursos dedicados a la implantación del modelo.

Algunas soluciones a este reto son: tener en cuenta las prioridades de los ejecutivos, usar equipos interdepartamentales, revisar y aprobar los nuevos procesos en un grupo, que incluya todos los niveles de gerencia de la empresa, para que tengan la oportunidad de analizar la integración y el efecto de los cambios propuestos. Así mismo, especificar un procedimiento a nivel de gerencia, para definir y revisar las políticas de la organización, establecer comités gerenciales que monitoreen periódicamente el desempeño de los nuevos procesos, e implantar desde el comienzo las prácticas genéricas, las cuales aseguran la institucionalización de los procesos en la organización.

\subsubsection{Difusión}

Este reto se relaciona con la administración del conocimiento generado por la implantación de los nuevos procesos y cuya responsabilidad recae en el EPG. Los síntomas de este reto se presentan cuando los grupos de innovación se aíslan, se enfrentan a los grupos que usan las prácticas tradicionales, crean algo que ya otro grupo utiliza, tratan de imponer prácticas, o caen en la arrogancia.

Este reto va más allá de establecer un sistema de información que permita almacenar y compartir la información de procesos, y apoyar la automatización de los mismos. Si bien la instalación de herramientas de apoyo para realizar los procesos del CMMI es difícil, lo es aún más, conseguir que todas las personas de la organización sean adiestradas en su uso y las utilicen de forma oportuna, eficiente y eficaz. Este reto también incluye la creación de una infraestructura de apoyo para las comunidades de práctica y la capacitación de todos los roles de la empresa, en sus nuevas rutinas y procedimientos. Todos necesitan saber a quién, a dónde acudir y que preguntar, antes de que puedan aprender de las experiencias de otros.

Además, está la problemática del manejo de las versiones de los procesos. Es lamentable cuando las personas utilizan un formato y luego la persona de aseguramiento de la calidad, lo rechaza porque no corresponde con la última versión aprobada. Al mismo tiempo, el EPG debe crear una cultura que fomente la investigación, cooperación y el compartir las ideas entre los diferentes roles de la empresa. Este reto también implica crear una capacidad de soporte al interior de sí misma y el desarrollo de entrenadores internos.

Algunas soluciones a este reto son: establecer una librería de procesos, con todos los formatos, procedimientos, ejemplos, lecciones aprendidas, entre otros. Además, instalar y adiestrar en el uso de las herramientas automatizadas de apoyo a los procesos. Así mismo, es necesario definir, planear y comunicar la estrategia de capacitación y soporte para las nuevas prácticas, como también, realizar talleres de explicación sobre la forma de medir, registrar y analizar el desempeño de los nuevos procesos. 


\subsubsection{Estrategia y propósito}

Cuando los grupos de innovación tienen éxito y logran mantener los cambios durante algún tiempo, invariablemente surgen nuevas aspiraciones e ideas sobre oportunidades de negocios. Esto se debe al aumento en las competencias, confianza, creatividad y reflexión, cuando se reconoce que el cambio es un estilo de vida. A medida que esto sucede, los grupos de innovación dedican más tiempo en cuestionar el propósito y los supuestos sobre la naturaleza del negocio. Si en el resto de la organización tiene poca capacidad de renovación y no presta una atención seria a las propuestas de mejora, se generará un vacío o brecha y los innovadores terminarán desilusionándose, se sienten frustrados porque no pueden usar su pensamiento estratégico al servicio de la empresa, la consecuencia es una pérdida de creatividad y pasión, disminuyendo la voluntad de la gente para comprometerse en nuevas iniciativas de cambio, o incluso retirándose de dichos grupos.

Algunas soluciones a este reto son: formalizar los ejercicios de planeación estratégica de forma sistemática, del mismo modo, definir y usar un procedimiento para reportar, analizar e implantar propuestas de mejora a los procesos actuales y desarrollar un mejor pensamiento estratégico.

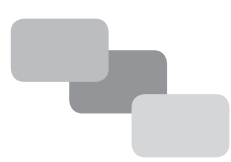

\section{CONCLUSIONES}

C uando una empresa considera implantar el Modelo CMMI, es importante tener en cuenta los siguientes elementos:

- Explicar a la gerencia la visión del modelo, de los niveles y de cada proceso, para que esta analice el impacto de los cambios y pueda comprometerse con la iniciativa.

- Iniciar la implantación del modelo solo cuando se obtenga el compromiso de la gerencia, explicándole a esta, el alcance de la iniciativa.

- Entrenar al EPG en la dinámica del cambio, pues el EPG debe actuar un nivel más del que se está implantando, es decir, EPG debe tener la cultura del nivel 2, cuando la empresa está en nivel 1 y decide implantar el nivel 2.

- Usar una metodología de implantación reconocida y probada, como es IDEAL.
- Definir una infraestructura de mejora (MSG, EPG, PATs, entre otros) desde el lanzamiento del proyecto, todos deben saber el rol que desempeñarán en la implantación, el grado de dedicación esperada y cuáles serán sus responsabilidades.

- No ahorrar en capacitación. La Ingeniería de Software es una disciplina amplia, con múltiples conceptos ya trabajados, para lo cual no hace sentido dedicar tiempo y recursos en inventar lo que ya esta creado.

- Convencer con el ejemplo. Al final, la implantación debe generar un cambio en los valores, supuestos y modelos mentales de las personas. No se puede hablar de algo, escribirlo en unos procedimientos, y actuar en sentido contrario.

- La implantación del CMMI es un proyecto, en consecuencia, se deben aplicar las prácticas de gerencia de proyectos. 
- Reconocer que la implantación del CMMI tomará tiempo y recursos y debe hacerse en paralelo con los proyectos del negocio y dentro del horario laborable, en consecuencia, se debe definir desde el inicio el manejo de los compromisos ya adquiridos, por las personas que participaran en la implantación. Esto puede requerir la contratación de recursos temporales, que liberen tiempo y permitan la dedicación y reflexión de los participantes.

- La implantación del CMMI debe tener un plan de proyecto conocido y disponible para consulta, de todas las personas de la organización. Todos deben saber que va ha pasar en los próximos meses, como aportarán y en que se beneficiarán.

- Los cambios de procesos deben hacerse de manera coordinada y de forma incremental, pues las personas no cambian de un día para otro.

- Es vital realizar logros tempranos, para que toda la organización adquiera confianza y sentido de logro, recordemos que Roma no se hizo en un solo día.
- Monitorear e informar periódicamente el avance del proyecto a toda la organización, pues esto aumenta el grado de compromiso en todos los niveles de la organización y convence a los incrédulos en la seriedad de la iniciativa.

- Identificar, aceptar y manejar la resistencia al cambio.

- Establecer y mantener una librería de procesos de fácil consulta, a toda la organización.

- Contratar asesores con experiencia conceptual y operacional reconocidas.

- Definir primero los procesos y luego investigar las herramientas automatizadas de apoyo. Así como el comprar un BMW no nos convierte en buenos conductores al instante, el tener una buena herramienta no hace que se institucionalicen los procesos y la nueva cultura organizacional. 


\section{REFERENCIAS BIBLIOGRÁFILAS}

Espejo, Schuhmann, W., Schwaninger, M. y Bilello U. (1996). Organizational Transformation and Learning. Chichester. Wiley. Cápitulo 6.

Chrissis, M., y Konrad, M., y Shrum S. (2003). CMMI: Guidelines for Process Integration and Product Improvement. Boston. Addison Wesley.

McFeeley, B. (1996). IDEAL Model Pittsburgh. Carnegie Mellon University,

Senge, P. (1999). The Dance of Change: The challenges of Sustaining Momentum in Learning Organization. London. Nicholas Breadley Publishing Limited.

Checkland, P.B. (1981). System Thinking, system practice. New York: Wiley.

Kan, S.H (2003). Metrics and models in software quality engineering. Pearson Education India.

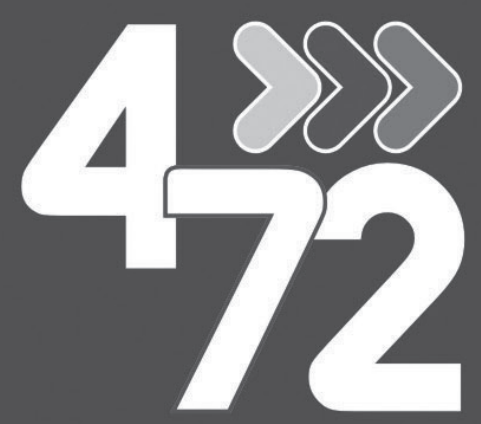

LA RED POSTAL DE COLOMBIA

$w w w .4-72 . c 0 m \cdot c 0$

$\checkmark$ Línea de Atención al Cliente Nacional 018000111210 